\title{
Análisis de la concentración del mercado del sector agrícola en el Ecuador. Período 2015 - 2019
}

\author{
Analysis of the market concentration of the \\ agricultural sector in Ecuador. Periodo 2015 - 2019
}

Armijos Bravo Manuela Inés Instituto Superior Tecnológico Argos, Ecuador marmijos@tecnologicoargos.edu.ec 


\title{
RESUMEN
}

El presente artículo estudia la competencia del mercado del sector agrícola en el Ecuador, específicamente de los subgrupos correspondientes a: A011 cultivo de plantas no perennes, y A012 cultivo de plantas perennes de la clasificación Industrial Internacional Uniforme para los años 2015 al 2019. En consiguiente, el objetivo que se plantea en el presente estudio es el de analizar la concentración del mercado del sector agrícola en el Ecuador empleando indicadores de concentración, tales como Cr4, Cr8, Herfindahl-Hirschman y número equivalente, para lo cual se ha utilizado las ventas netas en dólares reportadas por las empresas que operan en este sector, información que ha sido proporcionada por la Superintendencia de Compañías, Valores y Seguros del Ecuador para los años 2015-2019. Como principales conclusiones se puede mencionar que el mercado de los segmentos correspondientes a los cultivos de plantas no perennes, y cultivo de plantas perennes del 2015 al 2019 no se encuentra concentrado.

Palabras clave: sector agrícola, ingresos por ventas, cultivos de plantas no perennes; y cultivo de plantas perennes.

\begin{abstract}
This article studies the market competition of the agricultural sector in Ecuador, specifically of the subgroups corresponding to: A011 cultivation of non-perennial plants, and A012 cultivation of perennial plants of the International Standard Industrial Classification for the years 2015 to 2019. Consequently, the objective proposed in this study is to analyze the concentration of the agricultural sector market in Ecuador using concentration indicators, such as $\mathrm{Cr} 4, \mathrm{Cr} 8$, Herfindahl-Hirschman and the equivalent number, for which net sales have been used, in dollars, reported by the companies operating in this sector, information that has been provided by the Superintendency of Companies, Securities and Insurance of Ecuador for the years 2015-2019. As main conclusions, it can be mentioned that the market of the segments corresponding to the cultivation of non-perennial plants, and cultivation of perennial plants from 2015 to 2019 is not concentrated.
\end{abstract}

Keywords: agricultural sector, sales income, non-perennial plant crops; and cultivation of perennials. 


\section{INTRODUCCIÓN}

La Ley de Reforma Agraria en el Ecuador fue dictada en el año 1973, y desde allí nace la necesidad de realizar estudios de cómo ha sido la evolución de este sector; específicamente el presente análisis se enfoca en aquellas actividades correspondientes exclusivamente a cultivos de plantas no perennes subgrupo 011 y cultivos de plantas perennes subgrupo 012 de la clasificación Industrial Internacional Uniforme (CIIU). (Naciones Unidas, 2020)

Se considera importante realizar el presente estudio por algunas razones; primero, debido a que mide el grado de concentración de la participación en el mercado local de las actividades de cultivo de plantas no perennes y cultivo de plantas perennes en el Ecuador, puesto que estos rubros afectan la balanza de pagos del país, representando un valor aproximado del $20 \%$ de esta, acorde a la tabla 1 de este estudio.

Segundo, el desarrollo del sector agrícola ha sido considerado históricamente como uno de los sectores estratégicos, y parte neurálgica para el crecimiento económico del Ecuador, lamentablemente, el sector agrícola en el país no ha contado con programas sostenibles en el tiempo encaminados a su tecnificación; pues no solo se trata de procurar otorgar créditos con condiciones financieras diferenciadas para los agricultores, sino el contar con un ente técnico que los acompañe, asesore y encamine hacia una verdadera modernización del sector, de tal manera que a mediano y largo plazo el país sea capaz no solo de ofrecer al mercado internacional materia prima de gran calidad, sino de trabajar dicha materia con la finalidad de ofrecer productos con un valor agregado, para lograr una diferenciación positiva de sus competidores; para lo cual es importante que por intermedio del Ministerio de Agricultura y Ganadería el Gobierno del Ecuador se involucre de manera más comprometida con el sector agrícola.

Científicamente resulta de mucha utilidad realizar el presente estudio, puesto que el sector agropecuario en la región de América Latina es una actividad que presenta un riesgo financiero elevado en comparación con otro tipo de industrias, y quizás esa realidad contribuya en la existencia de concentración de mercado, pues ciertos cultivos son más susceptibles a los efectos climáticos, así como a la aparición de plagas, esto se debe en gran parte a que su rentabilidad no solo depende de la acción del hombre; sino que está supeditada a los embates de la naturaleza, situaciones que afectan en forma negativa a los productores e inversores en este mercado, ya que puede llegar a existir un alto índice de endeudamiento para adquirir materia prima y preparar la tierra para su siembra, pero al final del día no se puede predecir con certeza el azote de una plaga, o el grado de crudeza de algún fenómeno natural. Por tal motivo es importante que los países cuenten con planes y políticas de acción en materia de una adecuada gestión de prevención de riesgos e intervención en los casos de alguna catástrofe natural; pues hay que tomar en cuenta que la bonanza o rentabilidad del sector agrícola no solamente se mide en términos monetarios, sino que también está estrechamente ligada con la oportunidad al acceso de alimentos, hecho que deriva en la calidad de nutrición de la población (Arango, 2020). 
Adicionalmente las cinco principales contribuciones del sector agropecuario y forestal en la economía son:

a) Aporte del sector agropecuario al PIB. b) El sector agropecuario: aumento de las exportaciones y generaciones de divisas. c) El sector agropecuario y la generación del empleo. d) El sector agropecuario como generador de oferta de alimentos. e) El sector agropecuario y la formación de capital. De acuerdo a (Zúñiga, 2011, p.168)

Actualmente, a conocimiento de la autora, no existe una investigación enfocada en estos dos subgrupos que recoja datos y analice los índices de concentración de mercado para el período del presente estudio, 2015 al 2019. Sin embargo, existe amplia literatura que analiza la concentración de mercado de otros sectores económicos del Ecuador. Por ejemplo (Vite-Vera y Párraga-Fernández, 2019) analizan la concentración del mercado farmacéutico encontrando que la participación de mercado se concentra principalmente en las grandes empresas. (Camino-Mogro et al., 2017) encuentran evidencia que el mercado bancario en Ecuador posee una estructura oligopolista. Así mismo, (Horna et al., 2008) realizan un análisis de mercado del sector industrias manufactureras en base a CIIU 3 bajo un enfoque de concentración económica en el período 2000 - 2008 en el Ecuador, identificando aquellos sectores que son más abiertos a la competencia y los que se encuentran monopolizados. Finalmente (Solano et al., 2017) en la investigación que realizan sobre el análisis del entorno competitivo en el que operan las MIPYMES del sector manufacturero en Ecuador, llegan a la conclusión que cinco actividades manufactureras se encuentran altamente concentradas, estas son: elaboración de bebidas (C11), elaboración de productos de tabaco (C12), fabricación de coque y de productos de refinación del petróleo (C19), fabricación de productos de informática, electrónica y óptica (C26), y fabricación de vehículos automotores, remolques y semirremolques (C29).

La Constitución de la República del Ecuador, sección quinta, artículo 410, señala: "El Estado brindará a los agricultores y a las comunidades rurales apoyo para la conservación y restauración de los suelos, así como para el desarrollo de prácticas agrícolas que los protejan y promuevan la soberanía alimentaria" (Ley Orgánica 10/2008, de 20 de octubre, Constitución de la República del Ecuador) Con lo cual el Estado deja claro que dentro de sus competencias se encuentra la de brindar un adecuado soporte al sector agrícola para su buen desarrollo.

Las personas que laboran en la producción en el sector agrícola en el país pertenecen en su mayor parte al grupo de adultos, existe poco interés por parte de los jóvenes en incursionar en este sector. Es necesario mencionar que la instrucción formal de aquellas personas que se dedican a este sector en su gran mayoría solo posee un nivel de escolaridad primaria, situación que se ve acentuada por el hecho de que en el sector rural el $90 \%$ de los niños no acceden a la educación secundaria (Instituto Nacional de Estadística y Censos, 2008). 
Dentro de los productos primarios tradicionales que el Ecuador exporta se encuentran: banano y plátano, café y elaborados, camarón, cacao y elaborados; y atún y pescado. (Banco Central del Ecuador, 2020)

Tabla 1: Participación de los bienes del sector agrícola vs otros bienes en la Balanza de Pagos - 2019.

\begin{tabular}{|l|c|c|}
\hline BIENES & MONTO EN MILLONES DE DÓLARES & \% DE PARTICIPACIÓN \\
\hline Petrolera & $8,679.6$ & $51.00 \%$ \\
\hline No petroleras & \multicolumn{2}{|c|}{} \\
\hline Tradicionales & $8,337.8$ & $49.00 \%$ \\
\hline Banano y plátano & $3,295.2$ & $19.36 \%$ \\
\hline Café y elaborados & 80.2 & $0.47 \%$ \\
\hline Camarón & $3,890.5$ & $22.86 \%$ \\
\hline Cacao y elaborados & 763.9 & $4.49 \%$ \\
\hline Atún y pescado & 308.0 & $1.81 \%$ \\
\hline & $17,017.4$ & $100.00 \%$ \\
\hline
\end{tabular}

Fuente: Banco Central del Ecuador.

Para el año 2019, como se puede observar en la tabla 1, las exportaciones tradicionales representaron un $49 \%$ del total de las exportaciones acorde a la Balanza de Pagos; donde aquellas que pertenecen al sector agrícola, como lo es banano y plátano, alcanzaron solo un 19,36\% de representatividad, seguido por el cacao y elaborados con un $4,49 \%$ y finalmente café y elaborados con una mínima representación del 0,47\%. Esto deja de manifiesto la baja participación en las exportaciones del sector agrícola del país, la cual en su mayoría se concentran en banano y plátano.

El sector agrícola en el Ecuador está constituido por algunos subsectores relacionados con actividades de ganadería, silvicultura, cultivo de plantas, entre otras. Con respecto a la estructura del sector, de acuerdo a datos de la Superintendencia de Compañías, Valores y Seguros del Ecuador, la industria de cultivo de plantas no perennes tiene una participación del 11,58\%; y la industria de cultivo de plantas perennes posee una aportación mucho más interesante del 20,68\% dentro de los subsectores en la industria agrícola, ganadera, silvicultura y pesca, conforme se muestra en la tabla 2.

Tabla 2: Subsectores con mayor participación en la industria agrícola, ganadera, silvicultura y pesca.

\begin{tabular}{|l|l|l|r|l|}
\hline AÑo & CIIU & SUBSECTOR & INGRESO TOTAL & \% PARTICIPACIÓN \\
\hline 2019 & A011 & CULTIVO DE PLANTAS NO PERENNES. & $1,065,876,192.27$ & $11.58 \%$ \\
\hline 2019 & A012 & CULTIVO DE PLANTAS PERENNES. & $1,904,071,597.38$ & $20.68 \%$ \\
\hline 2019 & A013 & PROPAGACIÓN DE PLANTAS. & $50,866,655.07$ & $0.55 \%$ \\
\hline
\end{tabular}




\begin{tabular}{|c|c|c|c|c|}
\hline 2019 & $\mathrm{~A} 014$ & GANADERÍA. & $988,173,010.40$ & $10.73 \%$ \\
\hline 2019 & A015 & $\begin{array}{l}\text { CULTIVO DE PRODUCTOS AGRÍCOLAS } \\
\text { EN COMBINACIÓN CON LA CRÍA DE } \\
\text { ANIMALES (EXPLOTACIÓN MIXTA). }\end{array}$ & $832,501,439.63$ & $9.04 \%$ \\
\hline 2019 & A016 & $\begin{array}{l}\text { ACTIVIDADES DE APOYO A LA AGRI- } \\
\text { CULTURA Y LA GANADERİA Y ACTIVI- } \\
\text { DADES POSCOSECHA. }\end{array}$ & $701,175,177.52$ & $7.62 \%$ \\
\hline 2019 & A017 & $\begin{array}{l}\text { CAZA ORDINARIA, MEDIANTE TRAM- } \\
\text { PAS Y ACTIVIDADES DE SERVICIOS } \\
\text { CONEXAS. }\end{array}$ & $3,499,257.05$ & $0.04 \%$ \\
\hline 2019 & A021 & $\begin{array}{l}\text { SILVICULTURA Y OTRAS ACTIVIDA- } \\
\text { DES FORESTALES. }\end{array}$ & $69,500,658.43$ & $0.75 \%$ \\
\hline 2019 & A022 & EXTRACCIÓN DE MADERA. & $30,630,887.04$ & $0.33 \%$ \\
\hline 2019 & A024 & $\begin{array}{l}\text { SERVICIOS DE APOYO A LA SILVICUL- } \\
\text { TURA. }\end{array}$ & $4,261,008.77$ & $0.05 \%$ \\
\hline 2019 & A031 & PESCA. & $1,004,030,339.27$ & $10.91 \%$ \\
\hline 2019 & A032 & ACUICULTURA. & $2,550,860,574.94$ & $27.71 \%$ \\
\hline & & & $9,205,446,797.77$ & $100.00 \%$ \\
\hline
\end{tabular}

Fuente: Superintendencia de Compañías, Valores y Seguros.

Los principales productos que exporta el Ecuador son: banano, plátano, café, cacao y elaborados, a pesar de que produce muchos otros productos como: arroz, azúcar, mango, variedad de cereales, frutas y hortalizas, mismas que son comercializadas en su gran mayoría en el mercado interno.

En la tabla 3, se puede observar el ingreso por ventas netas del sector agrícola, que corresponde exclusivamente a la clasificación CIIU A011 "cultivo de plantas no perennes, y A012 cultivo de plantas perennes"; cabe señalar que los datos fueron obtenidos de la Superintendencia de Compañías, Valores y Seguros del Ecuador.

Tabla 3: Ingresos por ventas netas

\begin{tabular}{|l|l|c|}
\hline AÑOS & CIIU & INGRESO POR VENTAS NETAS \\
\hline 2015 & $\mathrm{~A} 011-\mathrm{A} 012$ & $3,978,412,329.73$ \\
\hline 2016 & $\mathrm{~A} 011-\mathrm{A} 012$ & $3,956,047,849.99$ \\
\hline 2017 & $\mathrm{~A} 011-\mathrm{A} 012$ & $4,546,311,658.16$ \\
\hline 2018 & $\mathrm{~A} 011-\mathrm{A} 012$ & $4,954,502,864.71$ \\
\hline 2019 & $\mathrm{~A} 011-\mathrm{A} 012$ & $3,299,681,864.75$ \\
\hline \multicolumn{2}{|l}{} & $20,734,956,567.34$ \\
\hline
\end{tabular}

Fuente: Superintendencia de Compañías, Valores y Seguros. 


\section{MATERIALES Y MÉTODOS}

Para realizar el presente análisis se utilizó datos administrativos provenientes de los estados financieros proporcionados por las empresas a la Superintendencia de Compañías, Valores y Seguros del Ecuador; la información fue segregada filtrando aquellas empresas que acorde al CIIU pertenecen a los grupos de A011 cultivo de plantas no perennes, y A012 cultivo de plantas perennes. La muestra utilizada se encuentra conformada por 985 empresas, de las cuales se procedió a trabajar con los valores de ventas netas en dólares reportadas durante los años de análisis, es decir del 2015 al 2019.

Para medir el nivel de concentración que existe en el sector agrícola ecuatoriano, para los cultivos de plantas no perennes, y cultivo de plantas perennes durante el período de análisis se utilizan los siguientes indicadores de concentración; índices $\mathrm{Cr} 4$ y Cr8, los cuales miden la participación en el mercado de las cuatro y ocho empresas más grandes de la industria (Beattie et al., 2003)

El ratio de concentración $(\mathrm{CRn})$ es la suma de las cuotas de mercado de las (m) empresas con mayor participación de los sectores que se están analizando, en consecuencia, es el valor que toma la curva de concentración para el punto m, (Núñez \& Pérez, 2001; Ginevičius \& Čirba, 2007) se define como sigue:

$$
C R_{m}=\sum_{i=1}^{m} S_{i}
$$

$$
C R_{4}=S_{1}+S_{2}+S_{3}+S_{4}=\frac{q_{1}}{Q}+\frac{q_{2}}{Q}+\frac{q_{3}}{Q}+\frac{q_{4}}{Q}
$$

Si el índice de concentración es 1 se asume que la industria tiene un comportamiento de monopolio en términos de cuota del mercado de acuerdo a la Superintendencia de Control del Poder de Mercado, 2016; el índice C4 es otra medida de concentración de mercado, la interpretación de sus resultados es:

- Menor a 33\% indica un mercado poco concentrado.

- Entre 33\% y 67\% indica un mercado moderadamente concentrado.

- Mayor a 67\% indica un mercado altamente concentrado.

El índice de Herfindahl-Hirschman, que se mide como la suma de los cuadrados de las cuotas de mercado de todas las empresas de una industria (U.S. Department of Justice and the Federal Trade C, 2020), se expresa matemáticamente de la siguiente forma:

$$
H H I=\sum_{i=1}^{m} S_{i}^{2}
$$


El departamento de Justicia y la Comisión Federal de Comercio de los Estados Unidos de América, U.S Department of Justice and the Federal Trade Commission (2010) por su nombre en inglés, ha definido las siguientes escalas del índice $\mathrm{HHI}$ :

HHI inferior a 0.01, el mercado es altamente competitivo.

HHI inferior a 0.15, el mercado no está concentrado.

HHI entre 0.15 y 0.25 , el mercado está moderadamente concentrado.

HHI mayor a 0.25, el mercado está altamente concentrado.

El cálculo del índice de Herfindahl-Hirschman también es útil para obtener el Número Equivalente, el cual muestra la cantidad de empresas con las que debería contar la industria dedicada a los cultivos de plantas no perennes, y cultivo de plantas perennes, para que la distribución de las empresas en los sectores analizados sea equitativa; la fórmula mediante la cual se la obtiene es:

$$
n^{*}=\frac{1}{H H I}
$$

\section{RESULTADOS Y DISCUSIÓN}

En la tabla 4 se observan los resultados después de aplicar los cuatro índices planteados al inicio de este análisis, con base en ellos se puede afirmar que la concentración del mercado para los subgrupos de A011 cultivo de plantas no perennes, y A012 cultivo de plantas perennes para cada uno de los años de análisis en el Ecuador; muestra un mercado no concentrado, durante los años 2015 y 2019 motivo del presente estudio.

Tabla 4: Índices de concentración

\begin{tabular}{|l|l|l|l|l|l|}
\hline AÑO & \multicolumn{1}{l}{ ÍNDICE } & \multicolumn{1}{l}{$\begin{array}{l}\text { INDICE } \\
\text { CR4 }\end{array}$} & \multicolumn{1}{l|}{ H-HI } & \multicolumn{1}{l|}{$\begin{array}{l}\text { NÚMERo } \\
\text { EQUIVALENTE }\end{array}$} \\
\hline 2015 & 0.19 & 0.26 & 0.02 & 54 & EL MERCADO NO ESTA CONCENTRADO \\
\hline 2016 & 0.18 & 0.24 & 0.02 & 57 & EL MERCADO NO ESTA CONCENTRADO \\
\hline 2017 & 0.17 & 0.25 & 0.02 & 65 & EL MERCADO NO ESTA CONCENTRADO \\
\hline 2018 & 0.15 & 0.22 & 0.01 & 83 & EL MERCADO ES ALTAMENTE COMPETITIVO \\
\hline 2019 & 0.24 & 0.32 & 0.03 & 35 & EL MERCADO NO ESTA CONCENTRADO \\
\hline
\end{tabular}

La industria del sector agrícola, en sus secciones de cultivos no perennes y perennes, acorde a los resultados encontrados en el presente análisis, se puede afirmar que no corresponde a un oligopolio, al contrario, muestra señales de ser un mercado competitivo, no concentrado. 
Como se puede observar en la tabla 4, los índices de concentración Cr4, Cr8 y H-HI para el año 2015 son de $0.19,0.26$ y 0.02 respectivamente lo cual sirve de evidencia para afirmar que el mercado de cultivo de plantas no perennes y perennes no se encontraba concentrado.

Para el año 2016 y 2017 los resultados dejan ver claramente que el mercado motivo del presente análisis no se encuentra concentrado, tal como lo revelan sus cifras en los índices $\mathrm{Cr} 4, \mathrm{Cr} 8$ y H-HI.

Acorde a los resultados de los índices Cr4, Cr8 y H-HI que corresponden al año 2018, 0.15, 0,22 y 0.01 se visualiza que el mercado es altamente competitivo, inclusive es muy superior al de los otros años de análisis; finalmente para el 2019 las cifras muestran un mercado no concentrado.

Los resultados de la presente investigación nos permiten determinar de forma clara que, durante los años de estudio, el mercado de los cultivos de plantas no perennes A011, y los cultivos de plantas perennes A012 ha sido competitivo en líneas generales y por lo tanto no se ha encontrado evidencia de indicios de concentración. Al igual que para el mercado manufacturero, en este sector las políticas públicas que genere, implemente e impulse el gobierno central por medio del Ministerio de Agricultura y Ganadería son trascendentales para generar un verdadero cambio positivo, que impulse a las medianas y pequeñas empresas a su tecnificación a mediano y largo plazo; genere condiciones crediticias óptimas para los productores; condiciones legales flexibles y sostenibles, es decir que no cambien con mucha frecuencia. Por medio del Ministerio de Transporte y Obras Públicas adecuar los caminos principales y secundarios para que los productores puedan transportar sus productos de manera oportuna; el Servicio Nacional de Gestión de Riesgos y Emergencias debe también involucrarse con la elaboración previa de planes de contingencia en los casos de sequía, o lluvias extremas que comprometan la integridad de los cultivos.

\section{CONCLUSIONES}

Como se ha mencionado en el apartado de Introducción a conocimiento de la autora no existe trabajo previo, que investigue el grado de concentración de los cultivos de plantas no perennes A011, y los cultivos de plantas perennes A012 durante los años que se ha realizado el presente estudio del 2015 al 2019.

Finalmente, como podemos observar en el cuadro 1 "Participación de los bienes del sector agrícola vs otros bienes en la Balanza de Pagos - 2019" las exportaciones tradicionales de cultivo de plantas no perennes y cultivo de plantas perennes representan en suma un aproximado del $20 \%$, por lo que es necesario que por medio de las diferentes carteras de estado e instituciones nombradas en este apartado colaboren de manera coordinada para lograr 
una verdadera transformación de los sectores abordados en el presente estudio que tiene el potencial de influir positivamente no solo en la balanza de pagos, sino también en el mejoramiento en el nivel de vida de todo el país.

El presente análisis busca incentivar investigaciones futuras en análisis de concentración de mercado dentro de los diferentes grupos que existen en el Sector Agrícola, y además complementarlo con otros indicadores de concentración de mercado, por ejemplo, un análisis econométrico.

Una limitación al presente trabajo, fue el no encontrar trabajos similares con los cuales se pueda contrastar o comparar los resultados finales de este artículo de investigación.

\section{REFERENCIAS BIBLIOGRÁFICAS}

Arango, M. (2019). El sector agropecuario de América Latina necesita más y mejores datos. Noviembre 2020, de Banco de Desarrollo de América Latina Sitio web: https://www.caf.com/es/conocimiento/visiones/2019/01/ el-sector-agropecuario-de-america-latina-necesita-mas-y-mejores-datos/.

Banco Central del Ecuador (2020). Boletín trimestral de la Balanza de Pagos del Ecuador. Recuperado de https://contenido.bce.fin.ec/documentos/Estadisticas/SectorExterno/BalanzaPagos/boletin73/indice.htm

Beattie, V., Goodacre, A., \& Fearnley, S. (2003). And then there were four: A study of UK audit market concentration-causes, consequences and the scope for market adjustment. Journal of Financial Regulation and Compliance, 11 (3), 250-265.

Ginevičius, R., \& Čirba, S. (2007, febrero 9). Determining market concentration. Journal of Business Economics and Management, VIII, 3-10. noviembre 24, 2020, De https://journals.vgtu.lt/index.php/JBEM/article/ view/7570/6537 Base de datos.

Horna, L., Guachamín, M., \& Osorio, N. (2009). Análisis de mercado del sector industrias manufactureras en base a CIIU 3 bajo un enfoque de concentración económica en el período 2000-2008 en el Ecuador. Revista Politécnica, 30, 230-243. 2020, noviembre 11, De https://bibdigital.epn.edu. ec/handle/15000/5543 Base de datos.

Instituto Nacional de Estadísticas y Censos. (2008). Estructura del Sector Agropecuario, según el enfoque de las características del productor agropecuario y de las unidades de producción agropecuaria. Ecuador: Instituto Nacional de Estadísticas y Censos. Recuperado de https://www. ecuadorencifras.gob.ec/documentos/web-inec/Bibliotecas/Estudios/Estudios_Economicos/Evolucion_de_la_indus_Alimen_Beb_2001-2006/Estruc_Sector_Agropecuario.pdf.

Ley Orgánica 10/2008, de 20 de octubre, Constitución de la República del Ecuador. Registro oficial 449, de 20 de octubre de 2008, https://www.gob. ec/sites/default/files/regulations/2020-06/CONSTITUCION\%202008.pdf. 
Ley Orgánica 10/2011, de 13 de octubre, Ley Orgánica de Regulación y Control del Poder de Mercado. Registro oficial 555, de 13 de octubre de 2011, https://www.oas.org/juridico/PDFs/mesicic4_ecu_org7.pdf

Naciones Unidas. (2009). Clasificación Industrial Internacional Uniforme de todas las actividades económicas (CIIU). noviembre 2020, de Departamento de Asuntos Económicos y Sociales Sitio web: https://unstats. un.org/unsd/publication/seriesm/seriesm 4rev4s.pdf.

Núñez, S., y Pérez, M. (2001). El grado de concentración en las ramas productivas de la economía española. noviembre 24, 2020, de Banco de España Sitio web: https://www.bde.es/f/webbde/SES/Secciones/Publicaciones/ PublicacionesSeriadas/DocumentosTrabajo/01/Fic/dt0113.pdf.

Solano, J., Camino, S., \& Alvarado, M. (2017, diciembre 18). Análisis del entorno competitivo en el que operan las Mipymes del sector manufacturero del Ecuador. Revista Empresarial, ICE-FEE-UCSG, 11, 54-63. 2020, noviembre 5, De https://www.researchgate.net/publication/325665578_ Analisis_del_entorno_competitivo_en_el_que_operan_las_mipymes_del_ sector_manufacturero_en_Ecuador Base de datos.

U.S. Department of Justice and the Federal Trade C. (2010). Horizontal Merger Guidelines. noviembre 18, 2020, de U.S. Department of Justice \& FTC Sitio web: https://www.justice.gov/atr/herfindahl-hirschman-index

Uzcátegui Sánchez, C., Camino Mogro, S., \& Moran Cruz, J. (2017, agosto 18). Estructura de mercado del sistema bancario ecuatoriano: concentración y poder de mercado. Revista Cumbres Vol $4 \mathrm{~N}^{\circ} 1$, pp. 9-16. 2020, noviembre 20, De https://investigacion.utmachala.edu.ec/revistas/index. php/Cumbres/article/view/271 Base de datos.

Vite-Vera, F. y Párraga-Fernández, J. (abril 2019). Análisis de la concentración de mercado del sector de elaboración de sustancias farmacéuticas en Ecuador. Período 2010-2017. Revista X-Pedientes Económicos, 3, 6-16. 2020, noviembre 20, De https://ojs.supercias.gob.ec/index.php/X-pedientes_Economicos/article/view/77/22 Base de datos.

Zúñiga, G. (2011). Contribuciones de la economía agrícola en el desarrollo local. En Texto Básico de Economía Agrícola su importancia para el desarrollo local sostenible (165-190). https://patriciacolon.files.wordpress. com/2015/01/libro-basico-economia-agricola.pdf: Universitaria, UNAMLeón. 
